\title{
Complete Chromosome-Scale Genome Sequence Resource for Sporisorium panici-leucophaei, the Causal Agent of Sourgrass Smut Disease
}

\author{
Gustavo Schiavone Crestana, ${ }^{1}$ Lucas Mitsuo Taniguti, ${ }^{2}$ Clesivan Pereira dos Santos, ${ }^{1}$ Juliana \\ Benevenuto, ${ }^{1}$ Paulo Cezar Ceresini, ${ }^{3}$ Giselle Carvalho, ${ }^{4}$ João Paulo Kitajima, ${ }^{2}$ Claudia Barros \\ Monteiro-Vitorello ${ }^{1, \dagger}$ \\ ${ }^{1}$ Genomics Group, Department of Genetics, University of São Paulo/Luiz de Queiroz College of \\ Agriculture (USP/ESALQ), Piracicaba SP 13418-900, Brazil \\ ${ }^{2}$ Mendelics Análise Genômica S.A., São Paulo SP 04013-000, Brazil \\ ${ }^{3}$ Molecular Phytopathology Lab, Plant Health, Rural Engineering and Soils Department, São Paulo State \\ University (UNESP), Ilha Solteira SP 15385-000, Brazil \\ ${ }^{4}$ SENAI Innovation Institute in Biomass, Três Lagoas MS 79640-250, Brazil
}

\section{Funding}

This work was supported by the following Brazilian institutions: i) Fundação de Amparo à Pesquisa do Estado de São Paulo grant numbers 2016/17545-8 and 2017/13268-2 (C. B. Monteiro-Vitorello) and fellowship 18/24028-5 (G. S. Crestana), ii) CNPq fellowships 144979/2018-7 (G. S. Crestana) and 302412/2018-2 (C. B. Monteiro-Vitorello), iii) National Council for the Improvement of Higher Education (CAPES) Finance Code 001, and iv) SENAI Innovation Institute in Biomass (G. Carvalho). Mendelics Análise Genômica provided support in the form of salary for authors L. M. Taniguti and J. P. Kitajima and had no role in study design, data collection, and analysis, decision to publish, or preparation of the manuscript.

\section{Keywords}

genome assembly, nanopore sequencing, plant-pathogen interaction, smut fungi, Sporisorium scitamineum
${ }^{\dagger}$ Corresponding author: C. B. Monteiro-Vitorello: cbmontei@usp.br

Current address for Juliana Benevenuto: Blueberry Breeding and Genomics Lab, Horticultural Science Department, University of Florida, Gainesville, FL 32611, U.S.A.

G. S. Crestana and L. M. Taniguti contributed equally to this work.

The author(s) declare no conflict of interest.

Accepted for publication 24 December 2020.
Copyright $\odot 2021$ The Author(s). This is an open access article distributed under the CC BY-NC-ND 4.0 International license. 

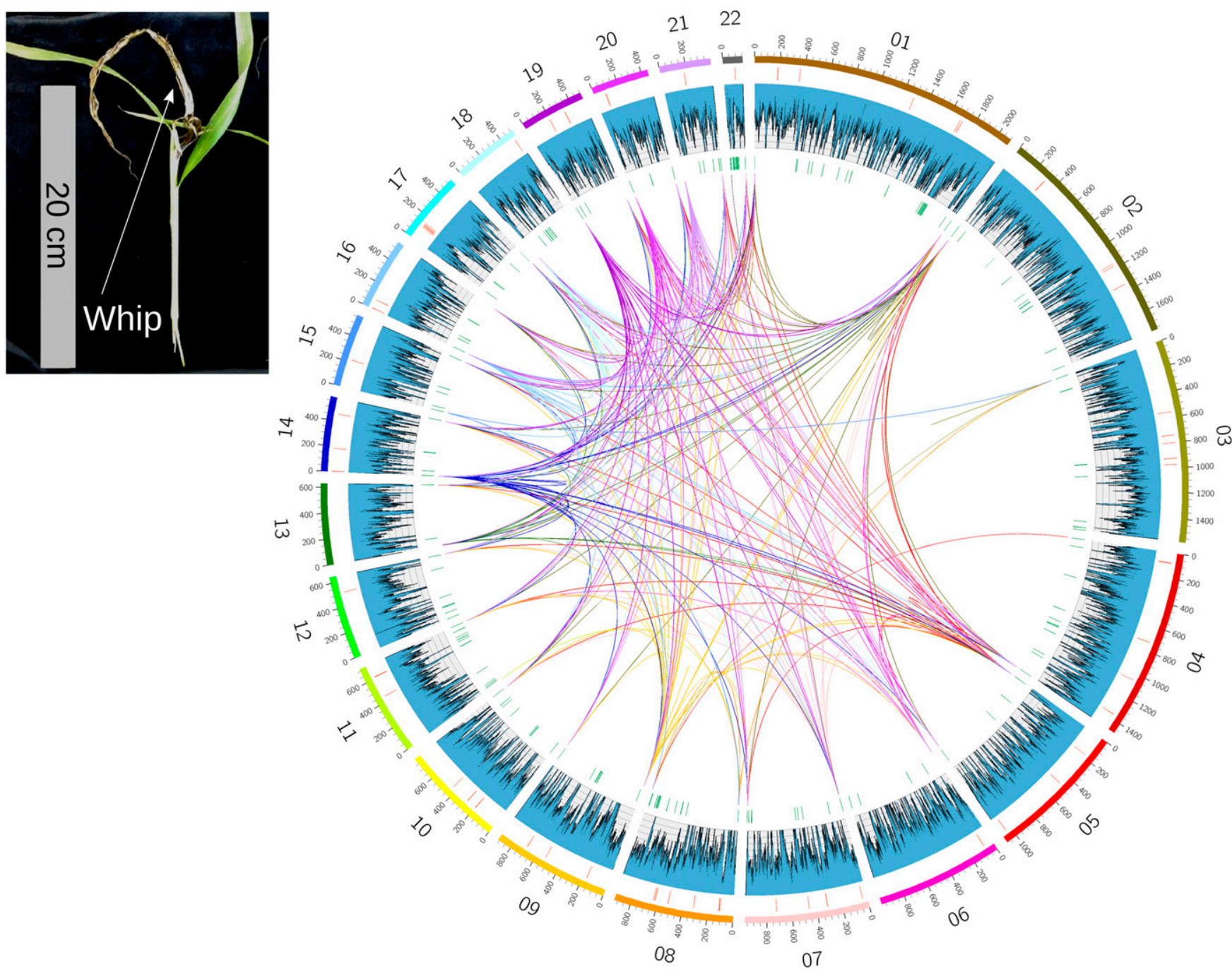

Fig. 1. Sourgrass smut disease symptom and the complete Sporisorium panici-leucophaei version 1 genome assembly. The small image shows the whip-shaped structure developed in an infected sourgrass plant from which we collected spores for fungal isolation. From the outside in, the genome image represents the following tracks: chromosomes in kilobase pairs of each chromosome; predictions of effectors in red tiles; a histogram with Illumina sequencing coverage ranging from 0 to 30x; green tiles representing repetitive elements; and colored links represent alignments of repetitive sequences ( $100 \mathrm{bp}<$ length $<500 \mathrm{bp}$ ) obtained by BLASTn (e-value < 1e-5 and identity of $95 \%$ ) on the first and last $500 \mathrm{bp}$ of each chromosome. Genome figure generated by Circos (Krzywinski et al. 2009).

establish host colonization. Common symptoms of smut diseases include the formation of tumors, abnormal floral components, and whip-like structure development (Benevenuto et al. 2018; Lo Presti et al. 2015).

Sporisorium panici-leucophaei (Bref.) M. Piepenbr., also known as Anthracocystis panici-leucophaeiand formerly described as Lundquistia panici-leucophaei (Cunnington et al. 2005; Piepenbring 1999; Stoll et al. 2005) infects several plant genera of the Poaceae family. However, sourgrass [Digitaria insularis (L.) Fedde], a well-known invasive weed species resistant to glyphosate, is considered the fungus primary host (Clayton and Renvoize 1986; Piatek 2006). The characteristic disease symptom is the growth of a whip-like structure from the culm apex, similar to those caused by another smut fungi infecting sugarcane, Sporisorium scitamineum (Taniguti et al. 2015). Studies on the pathogenicity and genomic features of this smut species are scarce. Here, we present a complete chromosome-scale genome sequence and features of the predicted secretome and effectorome of $S$. panici-leucophaei.

S. panici-leucophaei spores were isolated from infected sourgrass plants developing a whip-like structure (Fig. 1). Infected plants were collected in the Teaching, Research, and Extension Farm, FEPE, Universidade Estadual Paulista, Ilha Solteira Campus in the city of Selvíria, Mato Grosso do Sul, Brazil (approximate geographic coordinates: latitude 20²2' S 
Table 1. Summary of the genome sequencing, assembly and annotation statistics

\section{Feature}

Raw data (Nanopore) (Mbp)

Raw data (Illumina) (Mbp)

Assembly software

Genome (bp)

Total sequence coverage

Number of chromosomes

Largest chromosomes (bp)

Smallest chromosome (bp)

Mitochondrial genome (bp)

Mitochondrial contigs

Genome N50 (bp)

GC content (\%)

BUSCO completeness (genome mode) $(\%)$

BUSCO completeness (protein mode) (\%)

Repeat elements (\%)

Number of predicted protein-encoding genes

Number of predicted secreted proteins

Number of predicted effectors

Sporisorium panici-leucophaei

417
418
Canu
$18,915,934$
$32 \times$
22
$2,102,384$
152,803
78,051
1
969,070
53.99
97.7
97.2
2.71
6,402
388
68

and longitude $51^{\circ} 22^{\prime} \mathrm{W}$ ). Sequence analysis of the PCR-amplified internal transcribed spacer (ITS) region using universal ITS primers (Stoll et al. 2003) revealed $>99 \%$ identity with sequences of $S$. panici-leucophaei and A. panici-leucophaei available in GenBank (accession numbers AY344986, AY998102, and AY740035).

Yeast-like haploid cells germinated from a single spore (SPL10) were tested for matingtype (SPL10A) and were deposited at the Collection of Reference Fungi on Health Surveillance (CFRVS), FIOCRUZ, Instituto Oswaldo Cruz, under accession numbers 40436 and 40437. Haploid cells were grown as described by Benevenuto et al. (2016) for further DNA extraction and sequencing (Benevenuto et al. 2018). We used a hybrid assembly strategy by combining short reads from Illumina and long reads from Oxford Nanopore platforms to sequence and assemble the $S$. panici-leucophaei genome SPL10A.

High-molecular weight DNA was extracted using the Genomic-tip 20G kit according to manufacturer instructions for yeasts (Qiagen, Inc.). DNA integrity and quantity were checked on pulse-field gel electrophoresis (Taniguti et al. 2015) and were measured using a Qubit 2.0 Fluorometer (Invitrogen, ThermoFisher Scientific). The MinION sequencing library was prepared according to the Genomic DNA by Ligation kit (Oxford Nanopore Technologies), NEBNext Ultra II End-prep reaction/FFPE DNA repair (New England Biolabs), ligation buffer, NEBNext Quick T4 DNA ligase, and adapter mix, all according to manufacturer instructions. A sample of $1 \mu \mathrm{l}$ of the final library eluted in $15 \mu \mathrm{l}$ of Nanopore elution buffer was quantified and 5 to $50 \mathrm{fmol}$ was used for loading the flow cell with 45 pores available (MinION flow cells (R9.4.1) (Oxford Nanopore Technologies). The MinION device sequenced for $48 \mathrm{~h}$, and MinKNOW (MKE_1013_v1_revAR_11Apr2016) ran in mode base-calling real-time. We obtained a total of 69,189 reads ( $N 50=8,852 \mathrm{bp}$, maximum length $=89,048 \mathrm{bp}$, minimum length $=1,004 \mathrm{bp}$, average length $=6,016 \mathrm{bp}$ ) comprising $417 \mathrm{Mbp}$.

We then prepared a second DNA library using the Nextera DNA sample preparation workflow kit to obtain short paired-end sequences, using the MiSeq Illumina platform (2x $300 \mathrm{bp}$ ). Reads were analyzed using FASTQC (v.0.10.1) and Seqyclean (v.1.8.10) to filter low-quality sequences (Phred quality below 20 ). The resulting 715,363 reads generated approximately $418 \mathrm{Mbp}$ of DNA sequenced.

For the first step of genome assembling, we used Canu v1.8 (Koren et al. 2017) to assemble the long reads with the following parameters: genomeSize $=25 \mathrm{~m}$, correctedErrorRate $=$ 0.144 , and -nanopore-raw to specify the Nanopore reads (available on GitHub). All other parameters used were default set values, including minReadLength of $1 \mathrm{~kb}$. Second, we used the Illumina short reads to improve the assembly. BWA-MEM v0.7.17 was used to align short reads on the first raw assembly, and then, Pilon v1.23 (Walker et al. 2014) with the parameter "-fix bases,gaps" was used to correct potential misassemblies. Cross_match (Gordon et al. 1998) was used to close gaps in the scaffolds, generating an assembly with 23 contigs. Finally, we used Pilon again $(\times 2)$ to polish this final assembly. We covered each chromosome

450 / Molecular Plant-Microbe Interactions 
sequence with an average 15-fold of Illumina reads (Fig. 1) and approximately 17-fold of Nanopore reads (Table 1 ), resulting in a total 32 -fold coverage assembly.

Telomeric repeats were manually analyzed, searching for the canonical repeated sequence TTAGG (Benevenuto et al. 2016; Taniguti et al. 2015). Using RepeatModeler v1.0.11 and RepeatMasker v4.1.0 (Saha et al. 2008) (both available from the Institute for Systems Biology) containing the RepBaseRepeatMaskerEdition-20181026, we identified repetitive regions in the genome. Finally, BUSCO v4.1.2 (Simão et al. 2015) was applied to evaluate the genome assembly and gene prediction completeness, using the Basidiomycota database (basidiomycota_odb10.2019-11-20) as a reference.

The final complete chromosome-scale genome sequence of $S$. panici-leucophaei (version 1) comprised 18,915,934 nucleotides distributed in 22 chromosomes sequenced telomere-to-telomere (Fig. 1) and one mitochondrial DNA sequence of 78,051 bp (Table 1). The largest chromosome consisted of 2,102,384 bp (chromosome 1), whereas chromosome 22 had less than 155,000 bp (Table 1). The genome harbored around $3 \%$ repetitive sequences with no apparent biased distribution in the chromosomes (Fig. 1).

BUSCO v4.1.2 identified $97.7 \%$ completeness of the genome assembly and $97.2 \%$ of the protein prediction considering the 1,764 orthogroups in the reference database for Basidiomycota. Gene predictions accomplished with Augustus v3.3.3 (Stanke and Morgenstern 2005) (parameter-species = ustilago_maydis) and proteins of S. scitamineum (Taniguti et al. 2015) as extrinsic evidence, as described in section "alternate protocol 7 " of Hoff and Stanke. (2019), generated 6,402 protein-encoding genes.

We defined the predicted secretome of $S$. panici-leucophaei proteome following the pipeline: i) scanning proteins that presented a signal peptide with SignalP v5.0b (Almagro Armenteros et al. 2019), ii) selecting those that did not present a transmembrane domain with TMHMM V2.0c (Krogh et al. 2001), and iii) no endoplasmic reticulum retention sequence (PS00014) with PS-Scan (de Castro et al. 2006). Min (2010) showed that this strategy improved the accuracy of predictions. From a total of 6,402 predicted proteins, 388 proteins defined the fungus predicted secretome (Table 1). EffectorP 2.0 (Sperschneider et al. 2016) was used to predict the candidate effectors repertoire (effectorome) from the secretome, where approximately $17.5 \%$ (68 proteins) of all secreted proteins were considered candidate effectors (Fig. 1). To explore sequence identity to $S$. scitamineum genome, we used NUCmer from MUMmer v3.1 (Kurtz et al. 2004), with parameters --maxmatch -1 20, for global alignments. The comparison of $4.1 \mathrm{Mb}$ contiguous sequences between the two genomes resulted in approximately $87 \%$ identical nucleotides.

In the present study, we generated a complete genome assembly of S. panici-leucophaei and used it to predict the encoded proteins and the effectorome repertoire.

To the best of our knowledge, this is the first $S$. panici-leucophaei genome sequence available. We showed that a complete set of chromosomes (telomere-to-telomere) of a smut fungus could be achieved by combining long MinION nanopore and short MiSeq Illumina reads at low depth of coverage. We expect that the $S$. panici-leucophaei genome will provide a valuable resource for future comparative studies toward understanding pathogenicity in smuts.

Data availability. The complete chromosome-scale genome sequence of strain Sporisorium panici-leucophaei SPL10A has been deposited in GenBank under accession numbers CP060299 to CP060321 (BioProject: PRJNA648134; BioSample: SAMN15629615, Long Read Archive: SRR12314149/SRR12314150).

\section{Acknowledgments}

This Project was conducted in collaboration with São Paulo State University (UNESPCampus Ilha Solteira). Nonspecific permissions were required for the sampling of both spores and plants. The Project did not involve endangered or protected species.

\section{Author-Recommended Internet Resource}

GitHub leucophaei assembly: https://github.com//mtani/leucophaei-assembly

Vol. 34, No. 4, 2021 / 451 


\section{Literature Cited}

Almagro Armenteros, J. J., Tsirigos, K. D., Sønderby, C. K., Petersen, T. N., Winther, O., Brunak, S., von Heijne, G., and Nielsen, H. 2019. SignalP 5.0 improves signal peptide predictions using deep neural networks. Nat. Biotechnol. 37:420-423.

Bakkeren, G., Kämper, J., and Schirawski, J. 2008. Sex in smut fungi: Structure, function and evolution of mating-type complexes. Fungal Genet. Biol. 45 (Suppl 1):S15-S21.

Benevenuto, J., Longatto, D. P., Reis, G. V., Mielnichuk, N., Palhares, A. C., Carvalho, G., Saito, S., Quecine, M. C., Sanguino, A., Vieira, M. L. C., Camargo, L. E. A., Creste, S., and Monteiro-Vitorello, C. B. 2016. Molecular variability and genetic relationship among Brazilian strains of the sugarcane smut fungus. FEMS Microbiol. Lett. 363:fnw277.

Benevenuto, J., Teixeira-Silva, N. S., Kuramae, E. E., Croll, D., and Monteiro-Vitorello, C. B. 2018. Comparative genomics of smut pathogens: Insights from orphans and positively selected genes into host specialization. Front. Microbiol. 9:660.

Clayton, W. D., and Renvoize, S. A. 1986. Genera Graminum, grasses of the world. Kew Bull. 13:389.

Cunnington, J. H., Vánky, K., and Shivas, R. G. 2005. Lundquistia is a synonym of Sporisorium (Ustilaginomycetes). Mycol. Balc. (Sofia) 2:95-99.

de Castro, E., Sigrist, C. J., Gattiker, A., Bulliard, V., Langendijk-Genevaux, P. S., Gasteiger, E., Bairoch, A., and Hulo, N. 2006. ScanProsite: Detection of PROSITE signature matches and ProRule-associated functional and structural residues in proteins. Nucleic Acids Res. 34:W362-W365.

Quijano, C. D., Wichmann, F., Schlaich, T., Fammartino, A., Huckauf, J., Schmidt, K., Unger, C., Broer, I., and Sautter, C. 2016. KP4 to control Ustilago tritici in wheat: Enhanced greenhouse resistance to loose smut and changes in transcript abundance of pathogen related genes in infected KP4 plants. Biotechnol. Rep. (Amst.) 11:90-98.

Geiser, E., Wiebach, V., Wierckx, N., and Blank, L. M. 2014. Prospecting the biodiversity of the fungal family Ustilaginaceae for the production of value-added chemicals. Fungal Biol. Biotechnol. 1:2.

Gordon, D., Abajian, C., and Green, P. 1998. Consed: A graphical tool for sequence finishing. Genome Res. 8:195-202.

Hoff, K. J., and Stanke, M. 2019. Predicting genes in single genomes with augustus. Curr. Protoc. Bioinformatics 65:e57.

Horst, R. K. 2013. Smuts. Pages 373-379 in: Westcott's Plant Disease Handbook, 8th Ed., K. H. Ralph, ed. Springer Netherlands, Dordrecht, Netherlands.

Koren, S., Walenz, B. P., Berlin, K., Miller, J. R., Bergman, N. H., and Phillippy, A. M. 2017. Canu: Scalable and accurate long-read assembly via adaptive $k$-mer weighting and repeat separation. Genome Res. 27:722-736.

Krogh, A., Larsson, B., von Heijne, G., and Sonnhammer, E. L. 2001. Predicting transmembrane protein topology with a hidden Markov model: Application to complete genomes. J. Mol. Biol. 305:567-580.

Krzywinski, M., Schein, J., Birol, I., Connors, J., Gascoyne, R., Horsman, D., Jones, S. J., and Marra, M. A. 2009. Circos: An information aesthetic for comparative genomics. Genome Res. 19:1639-1645.
Kurtz, S., Phillippy, A., Delcher, A. L., Smoot, M., Shumway, M., Antonescu, C., and Salzberg, S. L. 2004. Versatile and open software for comparing large genomes. Genome Biol. 5:R12.

Lo Presti, L., Lanver, D., Schweizer, G., Tanaka, S., Liang, L., Tollot, M., Zuccaro, A., Reissmann, S., and Kahmann, R. 2015. Fungal effectors and plant susceptibility. Annu. Rev. Plant Biol. 66:513-545.

Min, X. J. 2010. Evaluation of computational methods for secreted protein prediction in different eukaryotes. J. Proteomics Bioinform. 3:1-5.

Piatek, M. 2006. Sporisorium kenyanum, a new smut fungus with long twisted sori on Setaria pallide-fusca in Kenya. Polish Botanical Journal 51:159164.

Piepenbring, M. 1999. New and poorly known smut fungi in Cuba. Mycol. Res. 103: 459-467.

Saha, S., Bridges, S., Magbanua, Z. V., and Peterson, D. G. 2008. Empirical comparison of ab initio repeat finding programs. Nucleic Acids Res. 36 : 2284-2294.

Simão, F. A., Waterhouse, R. M., loannidis, P., Kriventseva, E. V., and Zdobnov, E. M. 2015. BUSCO: Assessing genome assembly and annotation completeness with single-copy orthologs. Bioinformatics 31:3210-3212.

Sperschneider, J., Gardiner, D. M., Dodds, P. N., Tini, F., Covarelli, L., Singh, K. B., Manners, J. M., and Taylor, J. M. 2016. EffectorP: Predicting fungal effector proteins from secretomes using machine learning. New Phytol. 210:743761.

Stanke, M., and Morgenstern, B. 2005. AUGUSTUS: A web server for gene prediction in eukaryotes that allows user-defined constraints. Nucleic Acids Res. 33 : W465-W467.

Stoll, M., Begerow, D., and Oberwinkler, F. 2005. Molecular phylogeny of Ustilago, Sporisorium, and related taxa based on combined analyses of rDNA sequences. Mycol. Res. 109:342-356.

Stoll, M., Piepenbring, M., Begerow, D., and Oberwinkler, F. 2003. Molecular phylogeny of Ustilago and Sporisorium species (Basidiomycota, Ustilaginales) based on internal transcribed spacer (ITS) sequences. Can. J. Bot. 81:976984.

Sundar, A. R., Barnabas, E. L., Malathi, P., and Viswanathan, R. 2012. A mini-review on smut disease of sugarcane caused by Sporisorium scitamineum. Pages 107-128 in: Botany. M. John, ed. InTechOpen, London.

Taniguti, L. M., Schaker, P. D., Benevenuto, J., Peters, L. P., Carvalho, G., Palhares, A., Quecine, M. C., Nunes, F. R., Kmit, M. C., Wai, A., Hausner, G., Aitken, K. S., Berkman, P. J., Fraser, J. A., Moolhuijzen, P. M., Coutinho, L. L., Creste, S., Vieira, M. L. C., Kitajima, J. P., and Monteiro-Vitorello, C. B. 2015. Complete genome sequence of Sporisorium scitamineum and biotrophic interaction transcriptome with sugarcane. PLoS One 10:e0129318.

Walker, B. J., Abeel, T., Shea, T., Priest, M., Abouelliel, A., Sakthikumar, S., Cuomo, C. A., Zeng, Q., Wortman, J., Young, S. K., and Earl, A. M. 2014. Pilon: An integrated tool for comprehensive microbial variant detection and genome assembly improvement. PLoS One 9:e112963. 